\title{
Mutations to Nitrofurantoin and Nitrofurazone Resistance in Escherichia coli K12
}

\author{
By ANTHONY S. BREEZE* AND EMMANUEL E. OBASEIKI-EBOR† \\ Microbiology Laboratory, Department of Pharmacy, Heriot-Watt University, 79 Grassmarket, \\ Edinburgh EH1 2HJ, U.K.
}

(Received 16 June 1982)

The isolation and properties of nitrofurantoin- and nitrofurazone-resistant mutants have been compared. Nitrofurantoin-resistant mutants were easier to obtain and showed a higher resistance level. There was some cross-resistance at lower drug concentrations but not at higher levels. There was no difference in the UV resistance of most of the mutants obtained although a $r e c B$ strain, $A B 2470$, did yield nitrofurantoin-resistant mutants with increased UV resistance. This was however the only repair-defective strain which could be mutated to nitrofurantoin resistance. It is suggested that there is a mechanism for nitrofurantoin resistance in Escherichia coli $\mathrm{K} 12$ which does not confer resistance to nitrofurazone and which may be associated with the repair of damaged DNA. This mechanism is in addition to that which also confers resistance to nitrofurazone.

\section{INTRODUCTION}

The nitrofurans form a group of drugs with a wide spectrum of antimicrobial activity. Several members of the group are used clinically: furazolidone for bacterial enteritis, nitrofurazone for topical infections and nitrofurantoin as a urinary tract antiseptic.

The precise mode of action of the nitrofurans is still not clear but it appears that nitrofurantoin and nitrofurazone have similar effects upon bacteria (Asnis et al., 1952; McCalla et al., 1975, 1978). The compounds are activated by one or more nitrofuran reductases to form bactericidal end-products which bind to DNA and protein (McCalla et al., 1970; Tu \& McCalla, 1975) with consequent inhibition of nucleic acid synthesis (McCalla, 1964; Plant \& Edwards, 1976; Lu \& McCalla, 1978). Further evidence that the drugs have a direct effect on DNA was provided by Jenkins \& Bennett (1976), Hamilton-Miller et al. (1977) and Lu et al. (1979) working with DNA repair mutants that showed increased sensitivity to the nitrofurans.

The similarity of the mode of action of nitrofurantoin and nitrofurazone has given rise to the general assumption that the development of resistance to the two drugs is similar. Hence most studies on nitrofuran resistance have used nitrofurazone-resistant mutants (Asnis et al., 1952; McCalla et al., 1970, 1978).

The aim of the work reported here was to investigate the development of nitrofurantoin resistance in Escherichia coli $\mathrm{K} 12$ and to compare the properties of any mutants obtained with those of nitrofurazone-resistant mutants. We also attempted the induction of nitrofurantoin resistance in DNA repair-defective strains.

\section{METHODS}

Bacteria. With the exception of Clostridium sporogenes NCTC 532 which was used as an indicator of anaerobic conditions, the bacteria used in this work were all strains of Escherichia coli K12 and are listed in Table 1. The properties of resistant mutants are described in Table 2.

$\dagger$ Present address: Department of Pharmaceutical Microbiology, Faculty of Pharmacy, University of Benin, Benin City, Nigeria. 
Table 1. Escherichia coli strains used

\begin{tabular}{llll} 
Strain & Relevant genotype & \multicolumn{1}{c}{ Source } & \multicolumn{1}{c}{ Reference } \\
W3110 & thy F $^{-}$ & Laboratory collection & Bachmann (1972) \\
AB1157 & See reference & Laboratory collection & Bachmann (1972) \\
AB2470 & As AB1157 recB & M. H. L. Green* & \\
NFR402 & As AB1157 $n f s A$ & M. H. L. Green & McCalla et al. (1978) \\
NFR502 & As AB1157 $n f s A$ nfsB & M. H. L. Green & McCalla et al. (1978) \\
& M. L. Green, Cell Mutation Unit, University of Sussex, Brighton, Sussex BN1 9QG, U.K.
\end{tabular}

* M. H. L. Green, M.R.C. Cell Mutation Unit, University of Sussex, Brighton, Sussex BN1 9QG, U.K.

Media and culture conditions. Bacteria were maintained on nutrient agar (Oxoid) slopes at $4{ }^{\circ} \mathrm{C}$ and were routinely cultivated in nutrient broth no. 2 (Oxoid), on nutrient agar or Diagnostic Sensitivity Test (DST) agar (Oxoid). Iron sulphite agar (Oxoid) was used for the growth of $C$. sporogenes.

Antibacterial agents. Nitrofurantoin was purchased from Sigma and nitrofurazone from Koch-Light. Stock solutions were prepared weekly at $300 \mu \mathrm{g} \mathrm{ml}^{-1}$ and stored at room temperature in amber bottles.

Isolation of nitrofurantoin- and nitrofurazone-resistant mutants. Approximately $10^{7}$ cells of sensitive strains were spread on nutrient agar plates containing increasing concentrations of either nitrofurantoin or nitrofurazone and incubated at $37^{\circ} \mathrm{C}$ for $48 \mathrm{~h}$. Resistant colonies from the highest concentrations of drug were picked and subcultured. These clones were then spread in turn on to higher concentrations of the appropriate drug. The procedure was repeated until maximum resistance was obtained.

Determination of $M I C$. The MIC values of the two nitrofurans were determined by spotting drops containing about $10^{5}$ cells per $\mathrm{ml}$ of normal saline on to overdried nutrient or DST agar plates containing doubling concentrations of the agents. For determinations under anaerobic conditions, the plates were placed in anaerobic jars containing 'Gas-Paks' (Becton Dickinson). Anaerobiosis was indicated using 'Gas-Pak' disposable anaerobic indicator strips and confirmed by the growth of $C$. sporogenes on iron sulphite agar. All plates were incubated at $37^{\circ} \mathrm{C}$ for $48 \mathrm{~h}$.

Determination of $U V$ resistance. Approximately $10^{3}$ bacterial cells diluted in saline were spread on nutrient agar plates and exposed to UV light at $254 \mathrm{~nm}$ with an intensity of about $0.86 \mathrm{~J} \mathrm{~m}^{-2} \mathrm{~s}^{-1}$. The irradiated plates were incubated at $37{ }^{\circ} \mathrm{C}$ for $24 \mathrm{~h}$ and a $50 \%$ survival time calculated as the time taken to reduce the viable count by $50 \%$.

Determination of nitrofuran reduction by whole cell suspensions. A modification of the methods used by McCalla et al. $(1970,1978)$ was used. Washed cell suspensions in $0.067 \mathrm{M}$-phosphate buffer $(\mathrm{pH} \mathrm{7.2)}$ and 0.05 $\mathrm{M}$-glucose were diluted to a cell density which gave an absorbance of $0.42 \pm 0.01$ at $600 \mathrm{~nm}$ in a Pye-Unicam SP30 UV spectrophotometer. A sample of the cell suspension $(2.9 \mathrm{ml})$ was incubated in the spectrophotometer for about $10 \mathrm{~min}$ at $37^{\circ} \mathrm{C}$ then $0.1 \mathrm{ml}$ nitrofurantoin or nitrofurazone at $300 \mu \mathrm{g} \mathrm{ml}^{-1}$ was added and the rate of reduction observed as a decrease in absorbance at $372 \mathrm{~nm}$.

\section{RESULTS}

\section{Isolation of resistant mutants and determination of nitrofuran sensitivity}

The MIC of nitrofurantoin and nitrofurazone for sensitive strains was found to be similar at $6 \mu \mathrm{g} \mathrm{ml}^{-1}$. For most cells $5 \mu \mathrm{g} \mathrm{ml}^{-1}$ was inhibitory but prolonged incubation allowed the emergence of resistant mutants. Sensitivity under anaerobic conditions was invariably slightly greater than under aerobic conditions.

The mutants isolated are listed in Table 2. First level nitrofurantoin-resistant mutants were more frequently obtained than first level nitrofurazone-resistant mutants. Nitrofurantoinresistant mutants were isolated in four consecutive steps giving a 25 -fold increase in resistance compared to the sensitive parents. First level mutants, e.g. D1, were typically threefold more resistant than the wild-type with a further threefold increase in second level mutants. Third level mutants showed a total increase in resistance of 18 -fold and final level mutants, e.g. EE45, were twenty-five times more resistant than the wild-type. The frequency of mutants at each step was fairly low, about 10 colonies per plate for the first and second steps but with rather more, about 50 colonies per plate, for the third and fourth steps.

First level nitrofurazone-resistant mutants, e.g. E1, showed a fourfold increase in resistance and second level mutants a further twofold increase. Attempts to increase nitrofurazone resistance further were unsuccessful, since colonies isolated on higher concentrations of 
Table 2. Nitrofuran resistance levels and nitrofuran reductase activities of mutants derived from W3110 and $A B 1157$

\begin{tabular}{llccc} 
Strain & Parent & $\begin{array}{c}\text { MIC of } \\
\text { nitrofurantoin } \\
\left(\mu \mathrm{g} \mathrm{ml}^{-1}\right)\end{array}$ & $\begin{array}{c}\text { MIC of } \\
\text { nitrofurazone } \\
\left(\mu \mathrm{g} \mathrm{ml}^{-1}\right)\end{array}$ & $\begin{array}{c}\text { Reduction } \\
\text { rate* }^{*}\end{array}$ \\
W3110 & & 6 & 6 & 81 \\
AB1157 & & 6 & 6 & 90 \\
AB2470 & AB1157 & $0 \cdot 5$ & $0 \cdot 5$ & 90 \\
& \multicolumn{5}{c}{ Selected on nitrofurantoin } & \\
D1 & W3110 & 20 & 15 & 59 \\
D2 & D1 & 60 & 40 & 20 \\
D3 & D2 & 110 & 50 & 9 \\
EE45 & D3 & 150 & 60 & 10 \\
ED1 & E2 & 150 & 50 & 10 \\
ED2 & NFR502 & 150 & 50 & 9 \\
ED29 & AB2470 & 8 & 8 & 88 \\
& & Selected on nitrofurazone & & \\
E1 & W3110 & 25 & 25 & 36 \\
E2 & E1 & 60 & 50 & 9
\end{tabular}

\footnotetext{
* $\mathrm{nmol}$ nitrofurantoin reduced in $30 \mathrm{~min}$ by $2.9 \mathrm{ml}$ cell suspension having an absorbance of $0.42 \pm 0.01$ at $600 \mathrm{~nm}$. Each value is the mean of at least three separate determinations.
}

nitrofurazone reverted to second level resistance on subculture. Second level mutants, e.g. E2 and NFR502, could be taken to fourth level nitrofurantoin resistance in a single step by nitrofurantoin selection. There was, however, no associated increase in nitrofurazone resistance.

Nitrofurantoin-resistant mutants of AB1157 were also isolated and showed levels of resistance comparable to those of mutants developed from W3110. Nitrofuran-resistant mutants could not be isolated from strains carrying mutations (e.g. $\operatorname{rec} A, \operatorname{lex} A, u v r B$ ) that render them deficient in DNA repair. However a $\operatorname{rec} B$ strain, AB2470, yielded low level nitrofurantoinresistant mutants e.g. ED29 which showed a 16-fold increase in resistance (Table 2). Attempts to increase the nitrofurantoin-resistance level of ED29 resulted in the formation of non-viable filaments.

Under anaerobic conditions, high level nitrofurantoin-resistant mutants, e.g. EE45, were twoto threefold less resistant. Nitrofurazone-resistant mutants were also less resistant to nitrofurazone, and ED1 and ED2 similarly showed increased sensitivity to nitrofurantoin under anaerobic conditions. This phenomenon of increased sensitivity to nitrofurans under anaerobic conditions was observed by Asnis et al. (1952) and has been attributed by McCalla et al. (1975) to the oxygen sensitivity of nitrofuran reductase activity (reductase II).

First level mutants resistant to each nitrofuran showed cross-resistance to the other except that nitrofurazone-resistant mutants, e.g. E1, were more resistant to nitrofurantoin than nitrofurantoin-resistant mutants, e.g. D1, were to nitrofurazone. Similarly both the second level mutants D2 and E2 were more resistant to nitrofurantoin than to nitrofurazone; D3 and EE45 showed much higher resistance to nitrofurantoin than to nitrofurazone. Mutants NFR402 and 502 isolated in other laboratories also showed this difference with respect to the two nitrofurans. Interestingly, EE45, E2 and NFR502 had similar levels of resistance to nitrofurazone, but EE45 was much more resistant to nitrofurantoin.

\section{$U V$ resistance of nitrofurantoin-resistant mutants}

There was no difference in the UV resistance of nitrofurantoin-resistant mutants and that of their drug-sensitive parents except that ED29 was markedly more UV-resistant than its $r e c B$ parent (AB2470) having a $50 \%$ survival time of $8 \mathrm{~s}$ compared to $2.5 \mathrm{~s}$. 


\section{Reduction of nitrofurans by whole cell suspensions}

Sensitive strains reduced nitrofurantoin rapidly but first level mutants showed a decreased rate of reduction (Table 2). Second level resistant mutants had a further decreased reduction rate while third and fourth level mutants had very similar, low, levels of reductase activity (about $10 \%$ of the sensitive wild-type level). There were no significant differences in the reduction of nitrofurantoin and nitrofurazone by whole cell suspensions of nitrofurantoin-resistant mutants.

First level nitrofurazone-resistant mutants were partially defective in nitrofuran reductase, while second level mutants showed only a very low level of reductase activity (Table 2). There was no difference in nitrofurantoin reduction by whole cell suspensions of ED1 and E2 but ED2 suspensions had rather less activity than those of its parent NFR502. Mutants ED1 and ED2 were considerably more resistant to nitrofurantoin than their parents.

The DNA repair-defective mutants which had been found to be extremely sensitive to nitrofurans did not have increased nitrofuran reductase activity. Whole cell suspensions of these mutants reduced nitrofurantoin at a rate comparable to that of wild-type strains.

\section{DISCUSSION}

There were several differences in the isolation and the properties of nitrofurantoin- and nitrofurazone-resistant mutants. The former were easier to obtain and higher levels of resistance could be achieved. Moreover, nitrofurazone-resistant mutants mutate to higher levels of resistance to nitrofurantoin without increasing the level of nitrofurazone resistance. Up to the third level, increases in resistance were accompanied by decreased nitrofuran reductase activity but the changes were not the same for the two drugs. First level nitrofurantoin resistance was accompanied by a $30 \%$ loss of reductase activity as opposed to a $55 \%$ loss with first level nitrofurazone-resistant mutants. The second level nitrofurazone-resistant mutant $\mathrm{E} 2$ exhibited maximum nitrofurazone resistance and the lowest level of reductase activity. Third and fourth level nitrofurantoin-resistant mutants shared this low level of enzyme activity. In this work NFR502 was found to have a level of reductase activity comparable to D2. However, this level was reduced to that of E2 when NFR502 was mutated to higher nitrofurantoin resistance.

In the isolation of multi-step resistant mutants there is always the possibility that different combinations of mutations will give rise to different levels of resistance. As in this work McCalla et al. (1978) found, however, that resistance always occurred in discrete steps. In this respect it is fortuitous that spontaneous mutants are readily obtainable, thus avoiding the need for mutagenesis.

The reciprocal cross-resistance of mutants to nitrofurantoin and nitrofurazone at the lower levels was interesting in that first level nitrofurantoin-resistant mutants retained a higher level of reductase and were more sensitive to both nitrofurans. At all levels nitrofurazone was more inhibitory than nitrofurantoin. Even though resistance to the latter could be greatly increased as seen in EE45 and ED2, there was only a slight increase in nitrofurazone resistance. This may possibly be explained by the observation of $\mathrm{Lu} \& \mathrm{McCalla}(1978)$ that unreduced nitrofurazone can have an effect on metabolism.

McCalla et al. (1978) demonstrated clearly that maximum resistance to nitrofurazone was the result of mutations in two genes, $n f s A$ and $n f s B$ leading to the loss of reductase I activity. The observation of four resistance steps in this work suggests that there could be at least three genes involved in resistance to nitrofurantoin. However, the situation is complicated by observations that mutation to full nitrofurantoin resistance by strain D2 required two steps, i.e. through D3 and EE45, whereas mutation of NFR502 in a single step gave an equivalent level of nitrofurantoin resistance but no increase in nitrofurazone resistance.

Strains of $E$. coli defective in DNA repair are known to be very sensitive to the nitrofurans (Jenkins \& Bennett, 1976; Hamilton-Miller et al., 1977). We therefore examined the UV resistance of our mutants to investigate the possibility that enhanced repair of DNA damage might be a mechanism for resistance to nitrofurantoin. There was, however, no difference in UV resistance with the exception of ED29, a mutant of the recB strain AB2470. It was markedly more UV- and nitrofurantoin-resistant and yet retained full reductase activity. It is apparent 
therefore that enhanced repair can be associated with nitrofurantoin resistance in some strains. Clearly there must be another mechanism operating which confers resistance to nitrofurantoin but not nitrofurazone and may be associated with the repair of damaged DNA.

One of us (E. E. O-E) gratefully acknowledges the receipt of a postgraduate award from the University of Benin. We thank Dr M. H. L. Green for several bacterial cultures.

\section{REFERENCES}

AsNIs, R. E., COHEN, F. B. \& Gots, J. S. (1952). Studies on bacterial resistance to furacin. Antibiotics and Chemotherapy 2, 123-129.

BachmanN, F. J. (1972). Pedigrees of some mutant strains of Escherichia coli K-12. Bacteriological Reviews 36, 525-557.

HAMilton-Miller, J. M. T., KerRy, D. W., REYNOLDS, A. V. \& BRUMFITT, W. (1977). Two new bioassay techniques for nitrofurans: Bacteroides fragilis and $\mathrm{Rec}^{-}$Escherichia coli as indicator strains. Chemotherapy 23, 236-242.

JENKINS, S. T. \& BeNNETT, P. M. (1976). Effect of mutations in deoxyribonucleic acid repair pathways on the sensitivity of Escherichia coli $\mathrm{K}-12$ strains to nitrofurantoin. Journal of Bacteriology 125, 1214 1216.

LU, C. \& McCalla, D. R. (1978). Action of some nitrofuran derivatives on glucose metabolism, ATP levels, and macromolecule synthesis in Escherichia coli. Canadian Journal of Microbiology 24, 650-657.

LU, C., McCalla, D. R. \& BRYant, D. W. (1979). Action of nitrofurans on Escherichia coli. Mutation and induction and repair of daughter-strand gaps in DNA. Mutation Research 67, 133-144.

MCCALLA, D. R. (1964). Effects of some nitrofurans on DNA synthesis and prophage induction. Canadian Journal of Biochemistry 42, 1245-1247.

McCalla, D. R., Reuvers, A. \& Kaiser, C. (1970). Mode of action of nitrofurazone. Journal of Bacteriology 104, 1126-1134.

McCalla, D. R., Olive, P., Tu, Y. \& FaN, M. L. (1975). Nitrofurazone-reducing enzymes in $E$. coli and their role in drug activation in vivo. Canadian Journal of Microbiology 21, 1484-1491.

McCalla, D. R., Kaiser, C. \& Green, M. H. L. (1978). Genetics of nitrofurazone resistance in Escherichia coli. Journal of Bacteriology 133, 10-16.

Plant, C. W. \& EDWARDS, D. I. (1976). The effects of tinidazole, metronidazole and nitrofurazone on nucleic acid synthesis in Clostridium bifermentans. Journal of Antimicrobial Chemotherapy 2, 203-209.

TU, Y. \& MCCALLA, D. R. (1975). Effect of activated nitrofurans on DNA. Biochimica et biophysica acta 402, 142-149. 